\title{
History of the National Health Insurance Cost for Radiological Examinations and Value of the Radiological Report Fee
}

영상의학과 보험수가 역사와 판독료의 가치

Choong Wook Lee, MD* (D)

Department of Radiology and the Research Institute of Radiology, University of Ulsan College of Medicine, Asan Medical Center, Seoul, Korea

J Korean Soc Radiol 2020;81(5):1038-1052

https://doi.org/10.3348/jksr.2020.0123

The author discovered some errors in Tables 1, 4, 5, 6 and Supplementary Tables 4, 5, 6. The corrected table appears below.

Table 1. The First Standard of Medical Costs and Guidelines for the Medical Fee Estimation Established in 1977

\begin{tabular}{c|r|l|}
\hline $\begin{array}{c}\text { Category } \\
\text { Code }\end{array}$ & $\begin{array}{r}\text { No. of } \\
\text { Article }\end{array}$ & \multicolumn{1}{|c|}{ Category Content } \\
\hline A & 5 & Basic medical treatment \\
\hline B & 107 & Examination \\
\hline C & 11 & X-ray reading \& examination \\
\hline D & 3 & Medication \& prescription \\
\hline E & 14 & Injection \\
\hline F & 9 & Anesthesia \\
\hline G & 13 & Physical therapy \\
\hline H & 11 & Neuropsychiatric therapy \\
\hline I & 590 & Treatment \& surgery \\
\hline Total (9) & 763 & \\
\hline
\end{tabular}

\begin{tabular}{|c|c|c|}
\hline $\begin{array}{c}\text { Category } \\
\text { Code }\end{array}$ & Examination & Score \\
\hline \multirow{3}{*}{$c-1$} & Fluoroscopy reading & \\
\hline & a. Without contrast media & 85 \\
\hline & b. With contrast media & 113 \\
\hline \multirow{4}{*}{$c-2$} & General reading & \\
\hline & a. Examinations of Category ' $\mathrm{C}-3$ (a)' or ' $\mathrm{C}-5$ (a), (c) or (d)' & 62 \\
\hline & b. Examinations of Category ' $\mathrm{C}-4$ ' or ' $\mathrm{C}-5$ (c)' & 91 \\
\hline & c. Others & 34 \\
\hline \multirow{4}{*}{$c-3$} & Simple radiography & \\
\hline & a. Head, Thorax, Spine or Pelvis & 73 \\
\hline & b. Phalanx \& Carpal/Tarsal bones & 51 \\
\hline & c. Others & 62 \\
\hline \multirow{2}{*}{ C-4 } & Special radiography & \\
\hline & a. Tomography (1ea) & 298 \\
\hline \multirow{5}{*}{ C-5 } & Radiography using contrast media & \\
\hline & a. Digestive system & 142 \\
\hline & b. Heart or Vascular system (1ea) & 1249 \\
\hline & c. Myelography & 340 \\
\hline & d. Others & 170 \\
\hline
\end{tabular}

Adapted from Ministry of Health and Social Affairs. Notice No. 16 (3). 
Table 4. The Global RVUs (Sum of the Technical and Professional RVUs) and the Converted Price of Major Radiology Examinations Informed in 2000

\begin{tabular}{lrr}
\hline \multicolumn{1}{c}{ Examination } & RVUs & Converted Price (KRW) \\
\hline Simple radiography & 70.22 & 3890 \\
$\quad$ Head, chest, abdomen, spine & 496.75 & 27520 \\
Upper gastrointestinal series & & \\
CT & 1188.96 & 65870 \\
$\quad$ Head & 1583.12 & 87700 \\
Chest & 1595.43 & 88390 \\
Abdomen & 1583.69 & 87740 \\
Spine & & \\
*Add 10\% of the RVU for the examinations using contrast media & \\
*Add 30\% of the RVU for the phase 2 dynamic CT, phase 3 dynamic CT, & \\
3-dimension CT, CT arthrography, cine CT and CT cisternography & & \\
\hline
\end{tabular}

Adapted from Ministry of Health and Welfare. Notice No. 2000-67 (12).

$\mathrm{KRW}=$ Korean won, $\mathrm{RVU}=$ relative value unit

Table 5. Changes in RVUs of Major Radiology Examinations After the First Edition (2013) and the Second Edition (2020) of Relative Value Scales

\begin{tabular}{|c|c|c|c|}
\hline \multirow{2}{*}{ Examination } & \multicolumn{3}{|c|}{ RVUs } \\
\hline & 2007 & 2013 & 2020 \\
\hline \multicolumn{4}{|l|}{ Simple radiography } \\
\hline Chest & 70.22 & 79.67 & 75.53 \\
\hline Abdomen & 70.22 & 54.23 & 48.69 \\
\hline Upper gastrointestinal series & 496.75 & 484.55 & 451.25 \\
\hline \multicolumn{4}{|l|}{ CT } \\
\hline Brain (without contrast media) & 1188.96 & 916.93 & 773.87 \\
\hline Chest (with contrast media) & 1741.43 & 1466.55 & 1379.27 \\
\hline Abdomen \& pelvis (with contrast media) & 1754.97 & 1413.27 & 1379.27 \\
\hline Spine (without contrast media) & 1583.69 & 1201.34 & 1004.22 \\
\hline \multicolumn{4}{|l|}{ MRI: basic } \\
\hline Knee joint (general, without contrast media) & 3773.79 & 2819.05 & 2513.43 \\
\hline Lumbosacral spine (general, without contrast) & 3430.72 & 2627.86 & 2385.25 \\
\hline \multicolumn{4}{|l|}{ MRI: special } \\
\hline Diffusion & 2401.50 & 1787.98 & 1678.22 \\
\hline \multicolumn{4}{|l|}{ Angiography } \\
\hline Superior mesenteric arteriography & 1429.57 & 4058.68 & 4043.01 \\
\hline
\end{tabular}

Adapted from Health Insurance Review \& Assessment Service. The Benefit Package of National Health Insurance 2007, 2013, 2020 (19, 20, 22).

RVUs = relative value units 
Table 6. RVUs of the Major Ultrasonography Examinations in January 2020

\begin{tabular}{lcc}
\hline \multicolumn{1}{c}{ Examinations } & RVUs & Converted Price (KRW) \\
\hline Neck ultrasonography: thyroid, parathyroid & 769.83 & 58661 \\
Breast·axilla ultrasonography & 1037.52 & 79059 \\
Abdominal ultrasonography & & \\
Liver·GB·bile duct·spleen·pancreas & & 77849 \\
\hline 1) General & 1021.64 & 115608 \\
2) Detailed & 1517.17 & 71056 \\
Appendix & 932.49 & \\
Male genital ultrasonography & & 73447 \\
\hline Prostate·seminal esicle (transrectum) & 963.88 & \\
\hline
\end{tabular}

Adapted from Health Insurance Review \& Assessment Service. The Benefit Package of National Health Insurance 2020 (22).

$\mathrm{GB}=$ gallbladder, $\mathrm{KRW}=$ Korean won, $\mathrm{RVUs}=$ relative value units

Supplementary Table 4. 주요 영상의학검사의 촬영료와 판독료가 통합된 상대가치점수와 환산수가(2000. 12.)

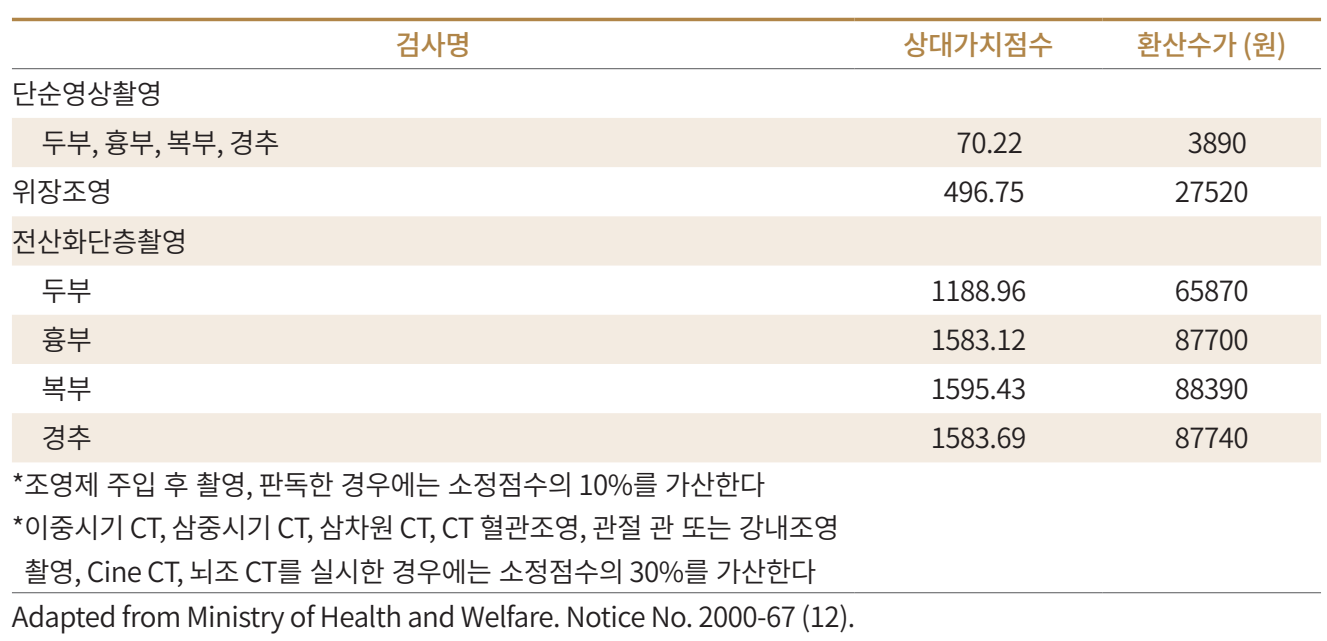


Supplementary Table 5. 1차 상대가치 개정(2013년)과 2차 상대가치 개정(2020년)에 따른 주요 영상의학검사의 상대가치점수 변화

\begin{tabular}{|c|c|c|c|}
\hline \multirow{2}{*}{ 검사명 } & \multicolumn{3}{|c|}{ 상대가치점수 } \\
\hline & 2007년 & 2013년 & 2020년 \\
\hline \multicolumn{4}{|l|}{ 단순영상촬영 } \\
\hline 흉부 & 70.22 & 79.67 & 75.53 \\
\hline 복부 & 70.22 & 54.23 & 48.69 \\
\hline 위장조영 & 496.75 & 484.55 & 451.25 \\
\hline \multicolumn{4}{|l|}{ 전산화단층촬영 } \\
\hline 두부(조영제를 사용하지 않는 경우) & 1188.96 & 916.93 & 773.87 \\
\hline 흉부(조영제를 사용하는 경우) & 1741.43 & 1466.55 & 1379.27 \\
\hline 복부(조영제를 사용하는 경우) & 1754.97 & 1413.27 & 1379.27 \\
\hline 척추(조영제를 사용하지 않는 경우) & 1583.69 & 1201.34 & 1004.22 \\
\hline \multicolumn{4}{|l|}{ 자기공명영상: 기본검사 } \\
\hline 슬관절(일반) & 3773.79 & 2819.05 & 2513.43 \\
\hline 요천추(일반) & 3430.72 & 2627.86 & 2385.25 \\
\hline \multicolumn{4}{|l|}{ 자기공명영상: 특수검사 } \\
\hline 확산 & 2401.50 & 1787.98 & 1678.22 \\
\hline \multicolumn{4}{|l|}{ 혈관조영촬영 } \\
\hline 상장간막동맥 & 1429.57 & 4058.68 & 4043.01 \\
\hline
\end{tabular}

Adapted from Health Insurance Review \& Assessment Service. The Benefit Package of National Health Insurance 2007, 2013, 2020 (19, 20, 22).

Supplementary Table 6. 주요 초음파 검사의 상대가치점수(2020년 1월 기준)

\begin{tabular}{lcc}
\hline \multicolumn{1}{c}{ 검사명 } & 상대가치점수 & 환산수가 (원) \\
\hline 경부 초음파: 감상선, 부갑상선 & 769.83 & 58661 \\
유방·액와부 초음파 & 1037.52 & 79059 \\
복부 초음파 & & \\
$\quad$ 간·담낭·담관·비장·췌장 & & \\
$\quad$ 1) 일반 & 1021.64 & 77849 \\
$\quad$ 2) 정밀 & 1517.17 & 115608 \\
$\quad$ 충수 & 932.49 & 71056 \\
남성생식기 초음파 & & \\
$\quad$ 전립선·정낭(경직장 검사) & 963.88 & 73447 \\
\hline
\end{tabular}

Adapted from Health Insurance Review \& Assessment Service. The Benefit Package of National Health Insurance 2020 (22). 